\title{
Cross-Species Amplification of Microsatellite Loci for the Endangered Conifer, Taxus chinensis var. mairei (Taxaceae)
}

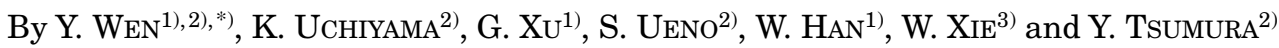

(Received 20 $0^{\text {th }}$ June 2012)

\begin{abstract}
Being an economical and endangered species, microsatellite markers of Taxus chinensis var. mairei were very limited. We have developed a set of microsatellite markers, which was benefit for future genetic analysis of this rare species. Polymorphic loci were developed from congeneric species by cross-species amplification methods, and new primers were redesigned to test for potential null alleles. 15 loci showed polymorphism. The number of alleles per locus varied from 2 to 23 tested in 48 individuals. The observed heterozygosity $(\mathrm{Ho})$ and expected heterozygosity $(\mathrm{He})$ values ranged form 0.000 to 0.854 and 0.082 to 0.827 , respectively. Newly redesigned primer confirmed that no null allele existed in most suspected loci. These microsatellite markers will be useful for future genetic analysis and conservation of this endangered species.
\end{abstract}

Key words: cross-amplification, microsatellites, Taxus chinensis var. mairei, population genetics, genetic diversity.

\section{Introduction}

Maire Yew (Taxus chinensis var. mairei) is an endemic Taxus species in China (ZHANG, et al., 1978). Its central distribution range consists of mainly scattered populations from the south of the Yellow river to the Nanling Mountain. Maire Yew has attracted much attention due to an anti-cancer drug that it contains, and due to the high aesthetic timber. However, long term over-exploitation has made this species become scarce and seriously threatened with extinction. In order to protect this rare species, the Chinese Government has listed Maire Yew among the first class protected plants since 1999 (The State of Council of the People's Republic of China, 1999), and several nature reserves have been set up in Southern China.

Conserving genetic diversity is one of the most important factors for threatened species. In order to conserve the genetic diversity, it has to be measured first. Compared with random fingerprinting approaches, microsatellites (simple sequence repeats, SSRs) are an ideal marker system for population genetics studies due to several advantages (JARNE and LAGODA, 1996). However, to develop new species-specific microsatellites demands knowledge about their flanking sequences

1) Central South University of Forestry and Technology, Changsha, Hunan 410004, China.

2) Forestry and Forest Products Research Institute, Tsukuba, Ibaraki 305-8687, Japan.

$\left.{ }^{3}\right)$ Guangxi University, Nanning, Guangxi 530004, China.

*) Author for correspondence: YAFENG WeN.

E-Mail: wenyafeng7107@163.com, wenyafeng@affrc.go.jp through traditional approaches, which is costly and time-consuming (ZANE et al., 2002; SQUIRRELL et al., 2003). One shortcut strategy is cross-species amplification to develop new species microsatellite markers (BARBARÁ et al., 2007), based on the conservation of microsatellites flanking regions in closely related species. This approach has been used in different studies, either to derive nuclear SSR (ALDRICH et al., 2003: PEAKALl et al., 1998) or cpSSR (WEISING and GARDNER, 1999; Chung and STAub, 2003) and EST-SSR marker (HEESACKER et al., 2008).

Until now, only a few studies focused on the genetic diversity of Maire Yew, by analyzing dominant markers, such as RAPD (ZHANG et al., 2003) and ISSR (LI et al., 2010). But no study was done based on microsatellites yet, due to the limited number of markers available. To the author's knowledge, microsatellite loci have been isolated from several Taxus species, including T. baccata (DUBREUIL et al., 2008, MAHMOODI et al., 2010), T. yunnanensis (MIAO et al., 2008), T. sumatrana (HUANG et al., 2008) and T. wallichiana (YANG et al., 2009). These loci are potential resources for Maire Yew in order to develop specific microsatellite markers. In the present study, we evaluated 68 candidate loci (including one mitochondrial marker) from congeneric species, and also tested 4 Maire Yew microsatellite loci developed by ZHOU et al (2009). 15 polymorphic markers were selected and their characterization were described.

\section{Materials and Methods}

In order to evaluate the polymorphism of the candidate microsatellite markers, 48 individual samples were selected from a wide range of Maire Yew's distributions in Southern China. DNA extraction from the silica dried leaves was carried out by a modified CTAB method (Tsumura et al., 1995). A total of 72 candidate loci were used to test PCR amplification and their polymorphism, in which 8 primer pairs were from T. yunnanensis (MIAO et al., 2008), 12 prime pairs were from T. sumatrana (HUANG et al., 2008), 10 primer pairs form T. wallichiana (YANG et al., 2009), 7 primer pairs from (DUBREUIL et al., 2008), 4 primer pairs from (ZHOU et al., 2009) and 31 primer pairs from (MAHMOODI et al., 2010). Some of the primers were end-modified to adapt post-PCR fluorescent labeling method (KonDo et al., 2000). For each of the primer pairs, 4 individuals were used to test whether they could be successfully amplified by PCR. PCR was performed in a $6.0 \mu \mathrm{l}$ volume according to QIAGEN $^{\circledR}$ Multiplex PCR kit instructions, containing $1 \times$ Multiplex PCR master mix (Qiagen), $0.2 \mu \mathrm{M}$ of forward and reverse primers, and 5-10 ng of template 
DNA. The PCR amplification conditions were: $15 \mathrm{~min}$ at $95^{\circ} \mathrm{C}$, then 30 cycles of $30 \mathrm{~s}$ at $94^{\circ} \mathrm{C}, 90 \mathrm{~s}$ at the primer pair's optimized annealing temperature (Table 1), and $60 \mathrm{~s}$ at $72^{\circ} \mathrm{C}$, followed by a $30 \mathrm{~min}$ extension step at $60^{\circ} \mathrm{C}$. The PCR products were electrophoretically separated on $2 \%$ agarose gels stained with ethidium bromide. For successfully amplifying loci, further singleplex PCR was carried out, in order to check their polymor- phism using 48 individuals. Primers were labeled at the 5' end with FAM or HEX fluorescent dyes (Applied Biosystems). PCR amplification system and conditions were the same as above. Genotypes were determined using an ABI3100 genetic analyzer and Liz 600 size standards. Population genetic parameters were estimated using GENALEX6.3 (PEAKALL and SMOUSE, 2006), including the number of alleles per locus $(\mathrm{Na})$, effective

Table 1. - Characterization of 15 polymorphic microsatellite loci in Maire Yew (Taxus chinensis var. mairei).

\begin{tabular}{|c|c|c|c|c|c|c|}
\hline Locus & $\begin{array}{c}\text { Primer } \\
\text { sequence (5'- 3') }\end{array}$ & Repeat motif & $\mathbf{T a} /{ }^{\circ} \mathbf{C}$ & $\begin{array}{c}\text { size } \\
\text { rang/bp }\end{array}$ & $\begin{array}{l}\text { GeneBan } \\
\text { accession }\end{array}$ & no \\
\hline \multirow[t]{2}{*}{ TY24 ${ }^{\mathrm{a}}$} & F: $<$ FAM $>$ GGG CTC GAC CTT TCT TCA A & $(\mathrm{AT})_{3}(\mathrm{ATCAT})_{10 \dagger}$ & 60 & $151-211$ & GU902801 & \\
\hline & R: GTT GGT CCT GTC CTC CCC ATA GAT & & & & & \\
\hline \multirow[t]{2}{*}{ TY0 $^{\mathrm{a}}$} & F: $<$ FAM $>$ CCA TAG CAT AGC AAG GGG TAA CC & $\begin{array}{c}(\mathrm{AGAT})_{4}(\mathrm{AG})_{2}(\mathrm{AGAT})_{4} \\
(\mathrm{AG})_{4}(\mathrm{AGAT})_{3}(\mathrm{AG})_{4}(\mathrm{AT})_{2}(\mathrm{AG})_{7}\end{array}$ & 60 & $457-471$ & GU902797 & \\
\hline & R: GTT CAT ACA GTC GAG GAG GGG GAG AAG & & & & & \\
\hline \multirow[t]{2}{*}{$\mathrm{TS}_{0} 3^{\mathrm{b}}$} & F: $<$ FAM $>$ CTC CTT CTA TAC TGC AAC CA & $(\mathrm{CA})_{5}$ & 60 & $264-288$ & & \\
\hline & R: GTT ATG TGT GTG TGA CTA GGG AA & & & & & \\
\hline \multirow[t]{2}{*}{ TY16 } & $\mathrm{F}:<\mathrm{FAM}>\mathrm{GGC}$ CCA CCC CCA CCA CA & $(\mathrm{CAGTT})_{5} \mathrm{CT}(\mathrm{AT})_{2}(\mathrm{AGAGGG})_{4}$ & 60 & $222-244$ & GU902800 & \\
\hline & R: GTT GGT GGT AGT TGG AGC CCC T & & & & & \\
\hline \multirow[t]{2}{*}{ TY0 $8^{\mathrm{a}}$} & F: $<$ FAM $>$ CCC CAT ATA TCC GGC CAT AC & $(\mathrm{TA})_{10}$ & 60 & $61-89$ & Gu902798 & \\
\hline & R: GTT GCA CTC CCA ACC CCA TCT CC & & & & & \\
\hline \multirow[t]{2}{*}{ TA114 } & F: $<$ FAM $>$ TCC CAG AAT ACT CCT TAT & $(\mathrm{AAG})_{2}$ & 57 & $210-213$ & FJ839828.1 & \\
\hline & R: GTT CTG GCT TCC TCT AGC ATT TCA T & & & & & \\
\hline \multirow[t]{2}{*}{$\mathrm{TG} 129^{\mathrm{c}}$} & F: $<$ FAM $>$ TGG TTG GCA GAA ATA GAG & $(\mathrm{AG})_{6} \mathrm{AT}(\mathrm{AG})_{6}$ & 57 & $207-222$ & FJ839820 & \\
\hline & R: GTT GGT CTG ATA ATC TGA GGG & & & & & \\
\hline \multirow[t]{2}{*}{$\mathrm{TG} 126^{\mathrm{c}}$} & F: $<$ FAM $>$ CAT ACT ACT ACA CTC CAC CTA & $(\mathrm{AC})_{9}$ & 57 & $296-324$ & FJ839821 & \\
\hline & R: GTT TAA TCT CAG CCG CTA AAC & & & & & \\
\hline \multirow[t]{2}{*}{$\mathrm{TG} 47^{\mathrm{C}}$} & F: $<$ FAM $>$ CGG AGA TTG TCC TCA TAC & $(\mathrm{TC})_{7}-(\mathrm{AC})_{8}$ & 57 & $365-377$ & FJ839823 & \\
\hline & R: GTT ACT TCT TCT GTC CCA CCC & & & & & \\
\hline \multirow[t]{2}{*}{$\mathrm{TS} 10^{\mathrm{b}}$} & F: $<$ FAM $>$ GGA CAC CAA GTT CCT TCT TGA & $(\mathrm{AG})_{9}$ & 60 & 194-208 & & \\
\hline & R: GTT TGG ATT CTC CAT TCC AAA TGA & & & & & \\
\hline \multirow[t]{2}{*}{$\mathrm{TB}^{\mathrm{d}} 6^{\mathrm{d}}$} & F: $<$ FAM $>$ CCC TAG GGT TGG TGG AAT TT & $(\mathrm{GT})_{14}$ & 60 & 149-219 & EF012585 & \\
\hline & R: GTT TGT GGG AAT CCA TTT AAG CA & & & & & \\
\hline \multirow[t]{2}{*}{$\mathrm{TC}^{\mathrm{e}}$} & F: $<$ HEX $>$ ATT ATG CTA GGC TCA ACT & $(\mathrm{CA})_{9}$ & 53 & $129-145$ & EU391178 & \\
\hline & R: GTT GTT TGG ATG TCC ATG TGA & & & & & \\
\hline \multirow[t]{2}{*}{$\mathrm{TC}^{\mathrm{e}}$} & F: $<$ FAM $>$ GCT ATG GAA TGA AGA ATC & $(\mathrm{CA})_{6 \dagger}$ & 53 & $167-175$ & EU391176 & \\
\hline & R: GTT CGT CTT TAC TTT CCG TGT & & & & & \\
\hline \multirow[t]{2}{*}{$\mathrm{TC} 4^{\mathrm{e}}$} & F: $<$ FAM $>$ GAA TGC TTC CCA CAA TAG & $(\mathrm{GT})_{9}$ & 53 & $135-145$ & EU391175 & \\
\hline & R: GTT AAA CAT GGT GGC TAC ACT & & & & & \\
\hline \multirow[t]{2}{*}{ TB $01^{* a}$} & F: $<$ FAM $>$ TGG GAG AGC AGA GCA GTG ATT TAT & $(\mathrm{ATCTG})_{7}$ & 60 & $397-432$ & AB029370 & \\
\hline & R: GTT ACT GAG TGG TAC GGT TGG TTG G & & & & & \\
\hline
\end{tabular}

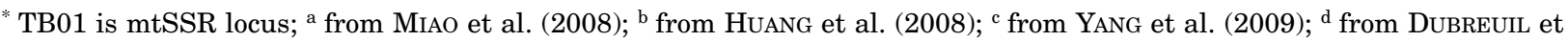
al. (2008); ${ }^{\mathrm{e}}$ from ZHOU et al. (2009).

Notes: For loci TS03 and TS10, DNA sequences were not submitted to GenBank by the authors. 
number of alleles per locus $(\mathrm{Ne})$, observed heterozygosity $(\mathrm{Ho})$, expected heterozygosity $(\mathrm{He})$ and fixation index $\left(F_{\text {IS }}\right)$. Hardy-Weinberg equilibrium $(H W E)$ and linkage disequilibrium $(L D)$ were assessed using GENEPOP (RAYMOND and ROUSSET, 1995) online version 4.0.10 (http://genepop.curtin.edu.au/index.html). Null alleles were detected by Micro-checker 2.2.3 (VAN OOSTERHOUT et al., 2004).

New primers were designed to confirm null alleles of the loci suspected by the results of Micro-checker 2.2.3. Direct sequencing of Maire Yew PCR products was according to the BigDye ${ }^{\circledR}$ Terminator v3.1 Cycle Sequencing Kit (Applied Biosystems). Ethanol purification products were run on an ABI3100 automatic sequencer. Forward and reverse nucleotide sequences of each locus were assembled by Sequencher 4.10, and then aligned using MEGA 5.05. New primers were designed for 7 loci by Primer3Plus online (http://www.bioinformatics.nl/cgi-bin/primer3plus/ primer3plus.cgi). PCR amplification and genotyping procedures with these new primers were the same as those of the original primers. According to the level of allele matching between the two primer sets, we tried to confirm the existence of null alleles at each locus.

\section{Results and Discussion}

Cross-species transfer success

Among the total of 72 candidate loci (one was a mtSSR marker), 38 (52.8\%) produced clear PCR fragment pat- terns (one or two bands), $3(4.2 \%)$ showed multiple bands (more than 2 bands); while 31 (43.0\%) did not produce any fragments. Out of 38 successfully amplifying loci, 15 (20.8\%) showed evidence for polymorphisms (Table 1); the others were monomorphic. The overall average cross-amplification success was $48.6 \%$, this data was less than conifer plants (66\%, BARBARÁ et al., 2007). When markers were compared in terms of species in origin, the rate of cross-amplification success and polymorphic loci were distinct among 4 congeneric species. Primers derived from T. yunnanensis, T. wallichiana and T. sumatrana had high success rate. Their success rate was $100 \%, 90 \%$ and $75 \%$, respectively, and polymorphic loci from these species were $66.7 \%, 40 \%$ and $16.7 \%$, respectively. However, those from $T$. baccata were $23.7 \%$ and $2.6 \%$. DUBREUIL et al. (2008) reported transferring $50 \%$ (4 of 8) microsatellite markers from T. sumatrana to T. baccata, and only one showed polymorphic banding pattern. MiAo et al. (2008) tested the transferability of $12 T$. sumatrana markers in T. yunnanensis, also only one loci worked.

According to several authors (PRIMMER et al., 1996; Guo et al., 2006; VINYALLONAGA et al., 2011) that crossamplification is except to work better for phylogenetically close species. Our data also support this idea. On the terms of phylogenetic relationship among species of Taxus, Marie Yew and T. yunnanensis has been recognized as a variety of T. wallichiana (FU et al., 1999); it had relatively far relationship with $T$. baccata (HAO et al., 2008).

Table 2. - Results of primer screening in 48 individuals of Taxus chinensis var. mairei.

\begin{tabular}{|c|c|c|c|c|c|c|c|c|c|}
\hline Locus & $N$ & $N a$ & $\mathrm{Ne}$ & Ho & $\mathrm{He}$ & Fis & $H W E$ & $\begin{array}{l}\text { Null alleles check } \\
\text { by Microchecker }\end{array}$ & $\begin{array}{l}\text { Predicted null } \\
\text { allele frequency }\end{array}$ \\
\hline TY24 & 48 & 11 & 5.519 & 0.021 & 0.827 & 0.975 & $* * *$ & Yes & 0.439 \\
\hline TY05 & 48 & 5 & 2.837 & 0.042 & 0.654 & 0.937 & $* * *$ & Yes & 0.368 \\
\hline TS03 & 48 & 3 & 1.986 & 0.417 & 0.502 & 0.171 & $\mathrm{~ns}$ & No & 0.053 \\
\hline TY16 & 48 & 6 & 2.698 & 0.000 & 0.636 & 1.000 & $* * *$ & Yes & 0.386 \\
\hline TY08 & 48 & 9 & 2.325 & 0.292 & 0.576 & 0.496 & $* * *$ & Yes & 0.177 \\
\hline TA114 & 48 & 2 & 1.280 & 0.042 & 0.221 & 0.813 & $* * *$ & Yes & 0.145 \\
\hline TG129 & 48 & 3 & 1.088 & 0.083 & 0.082 & -0.022 & ns & No & -0.002 \\
\hline TG126 & 48 & 11 & 3.990 & 0.542 & 0.757 & 0.287 & $* *$ & Yes & 0.119 \\
\hline TG47 & 48 & 7 & 3.313 & 0.625 & 0.705 & 0.115 & $* * *$ & No & 0.043 \\
\hline TS10 & 48 & 4 & 3.488 & 0.854 & 0.721 & -0.187 & $* * *$ & No & -0.082 \\
\hline TB86 & 48 & 23 & 4.934 & 0.500 & 0.806 & 0.382 & $* *$ & Yes & 0.165 \\
\hline $\mathrm{TC} 1$ & 48 & 6 & 2.012 & 0.458 & 0.508 & 0.099 & ns & No & 0.030 \\
\hline TC3 & 48 & 7 & 2.783 & 0.313 & 0.647 & 0.520 & $* * *$ & Yes & 0.200 \\
\hline TC4 & 48 & 6 & 2.312 & 0.083 & 0.573 & 0.856 & $* * *$ & Yes & 0.309 \\
\hline TB $01^{*}$ & 48 & 8 & & & & & & & \\
\hline
\end{tabular}

$N$ : number of samples, $\mathrm{Na}$ : number of alleles, $\mathrm{Ne}$ : effective number of alleles, $\mathrm{Ho}$ : observed heterozygosity, $H e$ : expected heterozygosity, Fis: fixation index; $H W E$ : Hardy-Weinberg equilibrium test.

ns: not significant departure from Hardy-Weinberg equilibrium $(H W E){ }^{*}{ }^{* *},{ }^{* * *}$ : significant departure from HardyWeinberg equilibrium ( $H W E)(P<0.01, P<0.001$, respectively).

${ }^{*}$ TB01 is mtSSR locus; ${ }^{\dagger}$ calculated using the method described by BRoOKFIELD (1996). 
Table 3. - New redesigned primers and the result of screening in 48 individuals of Taxus chinensis var. MAIREI.

\begin{tabular}{|c|c|c|c|c|c|c|}
\hline Locus & New primer sequence $\quad\left(5^{\prime}-3^{\prime}\right)$ & $\mathbf{T a} /{ }^{\circ} \mathbf{C}$ & $\begin{array}{l}\text { Size } \\
\text { range }\end{array}$ & $\begin{array}{c}\text { PCR } \\
\text { amplification }\end{array}$ & $\begin{array}{c}\text { Proportion of } \\
\text { matching genotypes }(\%)\end{array}$ & Accession no \\
\hline \multirow[t]{2}{*}{ N-TY24 } & F: GTT CTC TAC CCA TAG CGT TCA TTC AG & 60 & $115-175$ & Polymorphic & $48 / 48(100 \%)$ & AB725363 \\
\hline & R: ATT CTG TCC TCC CCA TAG ATC TCC & & & & & \\
\hline \multirow[t]{2}{*}{ N-TY05 } & F: $<$ FAM $>$ GGG GGA ACA TTC CTA CTC TAT CA & 60 & $273-288$ & Polymorphic & $48 / 48(100 \%)$ & AB725361 \\
\hline & R: GTT TAC AGT ACA GTT CGG AGT GAG & & & & & \\
\hline \multirow[t]{2}{*}{ N-TY16 } & F: GTG ACA GAT CTA CCA CAT CGT GA & 60 & $148-169$ & Polymorphic & $48 / 48(100 \%)$ & $\mathrm{AB} 725362$ \\
\hline & R: $<$ FAM $>$ TGG TAG TTG GAG CCC CTA TAC AT & & & & & \\
\hline \multirow[t]{2}{*}{ N-TC3 } & F: $<$ FAM $>$ TGC TAT GGA ATG AAG AAT CCA A & 55 & $157-165$ & Polymorphic & $39 / 45(86.7 \%)$ & AB725358 \\
\hline & R: GTT TCC GTG TGT GTT GTG TGT TTT & & & & & \\
\hline \multirow[t]{2}{*}{$\mathrm{N}-\mathrm{TC} 4$} & F: $<$ HEX $>$ ACA TGG TGG CTA CAC TAG AGC AC & 55 & $99-109$ & Polymorphic & $31 / 45(68.9 \%)$ & AB725359 \\
\hline & R: CAA CCT AGT GAG GAT CAT ACT TTC A & & & & & \\
\hline \multirow[t]{2}{*}{ N-TG126 } & F: ATT GCA TAG GGG GAG AGA TTC TAT T & 55 & 182 & Monomorphic & & $\mathrm{AB} 725360$ \\
\hline & R: GTT CTT ATC ACA GAG TGT CGC ACC A & & & & & \\
\hline \multirow[t]{2}{*}{ N-TA114 } & F: AAA TGT TCA GCC TCA AAA TCG & 55 & & No bands & & \\
\hline & R: GTT TGT TCT GGC TTC CTC TAG CA & & & & & \\
\hline
\end{tabular}

Notes: For loci TY08 and TB86, new primers were not redesigned due to sequence failure.

\section{Characterization of selected microsatellite loci}

Among the 15 polymorphic loci, the number of alleles per locus varied from 2 to 23 when tested on the 48 individuals. The observed heterozygosity $(\mathrm{Ho})$ and expected heterozygosity $(\mathrm{He})$ values ranged form 0.000 to 0.854 and 0.082 to 0.827 , respectively (Table 2). 10 loci were significantly deviated from Hardy-Weinberg equilibrium $(H W E)$ due to a large heterozygote deficiency $(P<0.01)$. Twenty-four out of 91 possible pairwise comparisons between the 14 nuclear microsatellite loci showed significant linkage disequilibrium $(p<0.05)$. Numerous factors might cause the deviation of Hardy-Weinberg equilibrium $(H W E)$, including inbreeding, null alleles and Wahlund effect. In our ongoing study among 26 populations using these nuclear markers, we have confirmed the influence of null alleles in most loci, which mean that inbreeding was the main reason for the large heterozygote deficiency and lead to deviation of HardyWeinberg equilibrium $(H W E)$. Another factor such as Wahlund effect might be one of the reasons, because 48 samples were selected from a wide range of Maire Yew's distributions in Southern China.

\section{Null allele's confirmation}

Micro-checker detection indicated no apparent evidence for scoring errors due to stuttering or large allele dropout in the 14 nuclear SSR loci. Possible null alleles were suggested by this software in 9 loci (Table 2). Our approach to design new primer for confirmation of null alleles suggested that no such null alleles existed in the 3 loci TY24, TY16 and TY05 (Table 3), as the agreement between the two primer sets was $100 \%$. For loci TC3 and TC4, the proportion of matching genotypes was
$86.7 \%$ and $68.9 \%$, respectively. This suggests there could be null alleles in these two loci. In our ongoing study among 26 populations using these 14 nuclear microsatellite loci (data not shown), TA114 and TG126 loci showed Hardy-Weinberg equilibrium $(H W E)$ in most populations, which we take as an indication that no null alleles are present in them. For loci TY08 and TB86, new primers were not redesigned due to sequencing failure, they need more cautious confirmation in a future study. We have used these 15 microsatellite markers in ongoing genetic analysis of Maire Yew, which will be benefit for the conservation of this endangered species.

\section{Acknowledgements}

This research was supported by the Special Fund for Forestry-scientific Research in the Public Interest of China (201104033), and also supported by Grant-in-Aid for JSPS Fellows (11F00787) and the Research Foundation of Education Bureau of Hunan Province China (09A103). The authors would like to thank Dr. HeINZE for critical reading of the manuscript and valuable suggestions.

\section{Reference}

Aldroch, P. R., M. Jagtap, C. H. Michler and S. J. RoMTERo (2003): Amplification of North American red oak microsatellite markers in European white oaks and Chinese chestnut. Silvae Genetica 52: 176-179.

Barbará, T., C. Palma-Silva, G. M. Paggi, F. Bered, M. F. FAY and C. LEXER (2007): Cross-species transfer of nuclear microsatellite markers: Potential and limitations. Molecular Ecology 16: 3759-3767. 
Chung, S. M and J. E. Staub (2003): The development and evaluation of consensus chloroplast primer pairs that possess highly variable sequence regions in a diverse array of plant taxa. Theoretical and Applied Genetics 107: 757-767.

Dubreuil, M., F. Sebastiani, M. Mayol, S. C. GonzalezMartinez, M. Riba and G. G. Vendramin (2008): Isolation and characterization of polymorphic nuclear microsatellite loci in Taxus baccata L. Conservation Genetics 9: 1665-1668.

FU, L. G., N. Li and R. R. Mill (1999): Flora of China (Vol. 4). Science Press, Beijing, Missouri Botanical Garden, St. Louis.

Guo, W., W. Wang, B. Zhou and T. Zhang (2006): Crossspecies transferability of $G$. arboreum-derived ESTSSRs in the diploid species of Gossypium. Theoretical and Applied Genetics 112: 1573-1581.

HaO, D.C., B. L. HuAng and L. YANG (2008): Phylogenetic relationships of the genus Taxus inferred from chloroplast intergenic spacer and nuclear coding DNA. Biological and pharamaceutical bulletin 31: 260-265.

Heesacker, A., V. K. Kishore, W. X. GaO, S. X. Tang, M. K. Judith, G. Alan, M. Marta, K. Alexander, M. M. RichaRD, L. ZHAO, H. R. LOREN and J. K. Steven (2008): SSRs and INDELs mined from the sunflower EST database: abundance, polymorphisms, and cross-taxa utility. Theoretical and Applied Genetics 117: 1021-1029.

HuAng, C. C., T. Y. ChIANG and T. W. Hsu (2008): Isolation and characterization of microsatellite loci in Taxus sumatrana (Taxaceae) using PCR-based isolation of microsatellite arrays (PIMA). Conservation Genetics 9: 471-473.

JARNE, P. and P. J. LAGODA (1996): Microsatellites, from molecules to populations and back. Trends in Ecology and Evolution 11: 424-429.

Kondo, H., T. TahiRa, H. Hayashi, K. Oshima and K. HAYASHI (2000): Microsatellite genotyping of post-PCR fluorescently labeled markers. Biotechniques 29: 868-872.

Li, N. W., S. A. He, X. C. Shu, Q. Wang and F. Peng (2010): Genetic diversity and structure analyses of wild and ex-situ conservation populations of Taxus chinensis var. mairei based on ISSR marker. Journal of Plant Resources and Environment 20: 25-30.

Mahmoodi, P., S. M. K. Nekouei, M. Mardi, S. M. Pirseyedi, M. R. Ghaffari, M. Ramroodi and B. A. SIAHSAR (2010): Isolation and characterization of new microsatellite marker in Taxus baccata L. Conservation Genetics Resources 2: 195-199.

Miao, Y. C., J. R. Su, Z. J. Zhang, H. Li, J. Luo and Y. P. ZHANG (2008): Isolation and characterization of microsatellite markers for the endangered Taxus yunnanensis. Conservation Genetics 9: 1683-1685.

Peakall, R. and P. E. SMouse (2006): GENALEX 6: Genetic analysis in Excel. Population genetic software for teaching and research. Molecular Ecology Notes 6: 288-295.
Peakall, R., S. Gilmore, W. Keys, M. Morgante and A. RAFALSKI (1998): Cross-species amplification of soybean (Glycinemax) simple sequence repeats (SSRs) within the genus and other legume genera: implications for the transferability of SSRs in plants. Molecular Biology and Evolution 15: 1275-1287.

Primmer, C. R., A. P. Møller and H. Ellegren (1996): A widerange survey of cross-species microsatellite amplification in birds. Molecular Ecology 5: 365-378.

RAYMOND, M and F. Rousset (1995): Genepop (Version1.2): Population genetics software for exact tests and ecumenicism. Journal of Heredity 86: 248-249.

Squirrell, J., P. M. Hollongsworth, M. Woodhead, J. Russell, A. J. Lowe, M. GiBby and W. Powell (2003): How much effort is required to isolate nuclear microsatellites from plants? Molecular Ecology 12: 1339-1348.

Tsumura, Y., K. Yoshimura, N. Tomaru and K. OHBA (1995): Molecular phylogeny of conifers using RFLP analysis of PCR-amplified specific chloroplast genes. Theoretical and Applied Geneticst 91: 1222-1236.

Van Oosterhout, C., W. F. Hutchinson, D. P. M. Wills and P. SHIPLEY (2004): MICRO CHECKER: software for identifying and correcting genotyping errors in microsatellite data. Molecular Ecology Notes 4: 535-538.

Vinyallonga, S. L., J. L. Alvarado, T. H. Constantinidis, A. SUSANNA and N. G. JACAS (2011): Microsatellite crossspecies amplification in the genus Centaurea (Compositae). Collectanea Botanica 30: 17-27.

WEISING, K. and R. C. GARDNER (1999): A set of conserved PCR primers for the analysis of simple sequence repeat polymorphisms in chloroplast genomes of dicotyledonous angiosperms. Genome 42: 9-19.

YANG, J. B., H. T. LI, D. Z. LI, J. LIU and L. M. GAO (2009): Isolation and characterization of microsatellite markers in the endangered species Taxus wallichiana using the FIASCO method. HortScience 44: 2043-2045.

ZANE, L, L. BARGELloni and T. PATARNELlo (2002): Strategies for microsatellite isolation: A review. Molecular Ecology 11: 1-16.

Zhang, H. Y., Y. Q. Chen and W. B. LiaO (2003): RAPD analysis of population genetic diversity of Taxus mairei. Acta Botanica Boreali-Occidentalia Sinica 23: 1994-1997.

ZHeng, W. J and L. G. FU (1978): Flora of China (Vol. 7). Science Press, Beijing.

Zhou, Y., G. P. Chen, Y. J. Su and Z. W. WANG (2009): Microsatellite loci from Taxus chinensis var. mairei (Taxaceae), an endangered and economically important tree species in China. Frontiers of Biology in China 4: 214-216. 